\title{
Identification And Validation of Pivotal Genes Related To Meniscus Senescence Based On Gene Expression Profiling Analysis And In Vivo And In Vitro Models Detection
}

\section{Ming Chen}

Department of Joint Surgery and Sports medicine, Zhongnan Hospital of Wuhan University

\section{Siqi Zhou}

Department of Joint Surgery and Sports medicine, Zhongnan Hospital of Wuhan University Huasong Shi

Department of Joint Surgery and Sports medicine, Zhongnan Hospital of Wuhan University Hanwen Gu

Department of Joint Surgery and Sports medicine, Zhongnan Hospital of Wuhan University Yinxian Wen

Department of Joint Surgery and Sports medicine, Zhongnan Hospital of Wuhan University Liaobin Chen ( $\square$ lbchen@whu.edu.cn )

Department of Joint Surgery and Sports medicine, Zhongnan Hospital of Wuhan University

\section{Research Article}

Keywords: Meniscus degeneration, Bioinformatics analysis, Senescence

Posted Date: June 25th, 2021

DOl: https://doi.org/10.21203/rs.3.rs-601815/v1

License: (c) (i) This work is licensed under a Creative Commons Attribution 4.0 International License. Read Full License 


\section{Abstract}

Background: The compositional change in the meniscus with aging would increase the tissue vulnerability of the meniscus, which would induce meniscus tearing. Here, we investigated the molecular mechanism of age-related meniscus degeneration with gene expression profiling analysis, and validate pivotal genes in vivo and in vitro models.

Methods: The GSE45233 dataset, including 6 elderly meniscus samples and 6 younger meniscus samples, was downloaded from the Gene Expression Omnibus (GEO) database. To screen the differential expression of mRNAs, identify the miRNAs targeting hub genes, and forecast the potentially toxic drugs, we completed a series of bioinformatics analyses, including functional and pathway enrichment, proteinprotein interaction network, hub genes screening, construction of a IncRNA-miRNA-mRNA network, and molecular docking of potential drugs. Furthermore, crucial genes were examined in human senescent menisci, mouse senescent meniscus tissues and mouse meniscus cells stimulated by IL-1ß.

Results: In total, the most significant 4 hub genes (RRM2, AURKB, CDK1, and TIMP1), 5 miRNAs (hsa-miR6810-5p, hsa-miR-4676-5p, hsa-miR-6877-5p, hsa-miR-8085, and hsa-miR-6133) that regulated such 4 hub genes, and potential toxic drugs (Cladribine, Danusertib, Barasertib, Riviciclib, and Dinaciclib) that had a targeting effect on these genes, were finally identified. Moreover, these hub genes were decreased in meniscus cells in vitro and meniscus tissues in vivo, which indicated that hub genes were related to meniscus senescence and could serve as potential biomarkers for age-related meniscus tearing.

Conclusions: In short, the integrated analysis of gene expression profile, co-expression network, and models detection identified pivotal genes, which elucidated the possible molecular basis underlying the senescence meniscus and also provided prognosis clues for early-onset age-related meniscus tearing.

\section{Introduction}

The meniscus is an important structure of knee joint, playing a vital role in load transmission, shock absorption, joint lubrication and nutrition, proprioception, and stability[1]. The position of the meniscus and its function in load transfer and shock absorption make the meniscus prone to traumatic and degenerative injuries. Therefore, the meniscus tearing is one of the most common intra-articular injuries of knee joint, and also one of the major causes of gonyalgia and lameness[2, 3]. Other studies have also suggested that meniscus tearing injury is not only one of the high-risk factors for inducing osteoarthritis $(\mathrm{OA})$, but also further aggravates the process of osteoarthritis, and ultimately affects people's basic daily activities $[4,5]$.

The population-based cohort study found that the age is a major risk factor for meniscus tearing, especially in people over 40 years old[6, 7]. Previous studies have shown that aging could cause compositional changes in the meniscus, which includes degeneration of collagens, cells, and proteoglycans[8, 9]. Meanwhile, these age-related tissue changes, such as aggregation of advanced glycation end products, thickening of collagen fibers, and mucoid degeneration, would increase the tissue 
vulnerability of the meniscus, which would eventually induce meniscus tearing[10]. Tsujii et al. found that age-related tissue homeostasis changes in the meniscus might be caused by senescence of meniscus cells[8]. However, there are few studies on meniscus aging, and also the specific molecular mechanism and key genes of meniscus cell senescence are still unclear[11, 12].

Bioinformatics is an emerging interdisciplinary field which combines molecular biology and information technology. It is of great importance to reveal that the differentially expressed genes (DEGs) are involved in molecular mechanisms, specific pathways, protein-protein interactions (PPI), and associations between disease and genes[13]. In order to study the molecular mechanism of age-related meniscus degeneration and further explore potential biological therapeutic targets, we carried out extensive bioinformatics methods to identify key DEGs and functional pathways in the aged meniscus tissues. Then, we constructed a IncRNA-miRNA-mRNA network and performed pharmacogenomics analysis to search potential miRNAs and drugs that may act on the screened key DEGs. Moreover, we further confirmed the expressions of these key DEGs in human senescent menisci, mouse aging meniscus tissues, and IL-1 $\beta$-stimulated mouse meniscus cells.

\section{Materials And Methods}

\section{Chemicals and reagents}

IL-1 $\beta$ was purchased from PeproTech Co., Ltd (Rocky, USA). The dulbecco's modified eagle medium-F12 (DMEM/F12) and phosphate buffer saline (PBS) were obtained from HyClone Co. (Logan, USA), while fetal bovine serum (FBS) was ordered from Gibco Co. (Detroit, USA). The TRIzol reagent was purchased from Invitrogen Co. (Carlsbad, USA). The SYBR Green dye and reverse transcription kits were purchased from Servicebio Co., Ltd (Wuhan, China). The antibodies for ribonucleotide reductase regulatory subunit M2 (RRM2, A5255), tissue inhibitor of metalloproteinases 1(TIMP1, A1389), and cyclin dependent kinase 1(CDK1, A0220) were ordered from ABclonal Technology Co., Ltd. (Wuhan, China). The aurora kinase B ( $A U R K B$, ab2254) and tissue inhibitor of metalloproteinases 1(TIMP1, ab216432) were purchased from Abcam plc. (Cambridge, UK). The SA- $\beta$-gal staining kits were obtained from Beyotime Co., Ltd. (Shanghai, China). The Annexin V-FITC/7-ADD apoptosis detection kits (Catalog Number: APK10448-F) were ordered from Sino Biological Co., Ltd. (Beijing, China). All primers were Tianyi Biotech Co., Ltd. (Wuhan, China).

\section{Data collection}

The GSE45233 dataset in Gene Expression Omnibus was downloaded for subsequent analysis. GSE45233 based on GPL10558 platform (Illumina HumanHT-12 V4.0 expression beadchip; Microarrays, Inc., San Diego, CA, USA) was demarcated by the age of 40, including 6 elderly meniscus samples and 6 younger meniscus samples, which were obtained from patients undergoing arthroscopic partial meniscectomy[14, 15].

\section{Identification of DEGs in elderly and younger samples}


We used the robust multiarray average algorithm to perform background correction and quartile data normalization on the GSE45233 dataset, and then identified DEGs associated with age by means of the Limma package in $\mathrm{R}$ language (version3.6.3). $P$ value $<0.05$ and $\log _{2}$ fold change $\left(\log _{2} \mathrm{FC}\right)<-1.5$ or $\log _{2} \mathrm{FC}>1.5$ were defined as the cutoff standard. The volcano plot and heatmap were completed in virtue of Ggplot2 and Pheatmap packages.

\section{KEGG and $\mathrm{GO}$ analysis annotate the functions of DEGs}

DAVID (version 6.8; https://david.ncifcrf.gov/) is an online bioinformatics database that integrates biological data and analysis tools to provide systematic and comprehensive biological annotation information for extensive lists of genes and proteins. The concept of Gene Ontology (GO) is widely used in the field of bioinformatics, which contains three notions of functional information: biological processes (BP), cellular components (CC), and molecular functions (MF). Currently, Kyoto Encyclopedia of Genes and Genomes (KEGG; https://www.kegg.jp/) is a database that integrates genomic, chemical, and system functional information for signaling pathway analysis. We employed the DAVID online tool to perform $\mathrm{GO}$ and KEGG analysis of DEGs.

\section{Construction of the PPI network}

STRING (Version11.0; https://string-db.org/), a search tool for interacting genes/proteins, was applied for predicting the PPI network and discovering the possible relationships. Cytoscape (version 3.8.0; https://cytoscape.org/), a powerful network building software, was used to establish the PPI network.

\section{Identification and analysis of significant hub genes}

The hub genes were screened through the PPI network utilizing the Cytoscape plugin cytoHubba. Since the singlular algorithm is sometimes biased, we employed a four-fold algorithm to collectively recognize hub genes, and these algorithms were adopted respectively. The algorithms used to recognize hub genes include Edge Percolated Component (EPC), Degree, Maximal Clique Centrality (MCC), and Maximum Neighborhood Component (MNC). Then the common hub genes were obtained through the intersection of Venn diagram websites (http://bioinformatics.psb.ugent.be/webtools/Venn). Metascape, as a forceful gene function analysis and annotation tools, was applied for batch enrichment analysis and network construction of genes and proteins to understand their functions. The common hub genes were further analysed through Metascape analysis.

\section{Construction of IncRNA-miRNA-mRNA network}

miRWalk (http://mirwalk.umm.uni-heidelberg.de/), as a bioinformatics suit to forecast miRNA-mRNA interactions, could predict miRNAs of the common Hub gene and then obtain the miRNA-mRNA interaction pairs with the score >0.9.[16] The DIANA-miRPath (https://www.microrna.gr/miRPathv3) is an online tool dedicated to assessing the regulatory role of miRNAs and forecasting relevant regulation pathways. The DIANA-LncBase (http://carolina.imis.athena-innovation.gr/diana_tools/) was conducted to search the IncRNAs targeting at miRNAs. In the end, a IncRNA-miRNA-mRNA regulatory network was accomplished by means of Cytoscape software. 


\section{Pharmacogenomics analysis of hub genes}

The Pharmacogenomics and Drug-Gene Interaction database (DGldb, http://dgidb.org/) work on collecting and categorizing information about the relationship between genetic variation and drug response. In this research, the network database was devoted to forecast drugs that might target at hub genes. Then, we downloaded 2D structures of small molecule drugs from the PubChem database (https://pubchem.ncbi.nlm.nih.gov/) before being prepared for docking. The software PyMol (version 2.4; https://pymol.org/2/) was applied to determine the lowest energy conformations for docking. With the help of AutoDock software (Version 4.2.6; http://autodock.scripps.edu/), we completed the flexible molecular docking between the macromolecular proteins and the drugs to determine the relative position and orientation in the process of a ligand binding to a receptor. Finally, the images were captured from software PyMoL.

\section{Human/Mouse meniscus tissues from normal and aging knees}

Human meniscus tissues were obtained from 12 patients undergoing arthroscopic partial meniscectomy, including 6 patients older than 40 years and 6 patients younger than 40 years. This human research protocol was authorized by the Medical Ethics Committees (approval number: 2019K-K011). All patients have signed informed consent. Animal protocols used in this study were approved by the Animal Welfare Committee (License number: 14016). Mouse meniscus tissues were collected from specific pathogen free (SPF) C57BL/6J male mice [NO.2020-0018, license number: SCXK (Hubei), certification number:42000600040335] purchasing from the Experimental Center of the Hubei Medical Scientific Academy (Wuhan, China) of two different ages, namely 3 months old $(n=6)$ and 18 months old $(n=6)$. All animals in this experiment were housed in a clean environment under a $12 \mathrm{~h}$ light/dark cycle for 1 week, where standard laboratory chow and water were freely consumed. Then, after the mice were anesthetized by intraperitoneal injection of $1 \%$ pentobarbital $(60 \mathrm{mg} / \mathrm{kg})$, the mice were euthanatized by spinal dislocation and the femurs were collected for subsequent detection. We provided an ARRIVE checklist in the Supplementary Material to reveal that the ARRIVE guidelines were adhered to in this research.

\section{Histological analysis in the meniscus tissues}

After fixation with $4 \%$ paraformaldehyde for 2 days, the entire mouse knee joints and human meniscus were decalcified, dehydrated and embedded in paraffin. The sections of mouse knee joints were stained with Safranin-O Fast Green and Toloniumchloride Blue to observe histopathological changes.

For immunohistochemistry analysis, knee joint sections were deparaffinized, hydrated, antigen retrieval, and blocked in 3\% BSA (Servicebio, Wuhan, China) at room temperature for $1 \mathrm{~h}$. Then, the sections incubated with primary antibodies for $A U R K B$ (1:200, dilution), CDK1 (1:100, dilution), RRM2 (1:100, dilution), and $\operatorname{TIMP1} 11: 250$, dilution, Abcam) overnight at $4^{\circ} \mathrm{C}$, followed by incubating with biotinylated secondary antibody (1:100 dilution). Finally, after the sections incubating with an avidin-biotinylated HRP 
complex solution, these were immersed in diaminobenzidine (DAB) to reveal peroxidase activity. Observations and photographs were taken under the Photo Imaging System (Nikon H550S, Japan). For immunohistochemical-negative controls, the primary antibodies were replaced by non-immunized IgG in immunostaining. Then, we measured the mean optical density (MOD) of 5 random fields in each section using Image Pro-plus (version 6.0) to determine the dyeing intensity. The percentage of positive cells in each field was the ratio of the number of positive cells to the total number of meniscus cells in the corresponding region.

\section{SA- $\beta$-gal staining}

The mouse meniscus cells were derived from meniscus tissues of SPF 3-month-old C57BL/6J mice. In brief, the full thickness meniscus tissues were cut into pieces, and digested with serum-free DMEM/antibiotics containing collagenase $D$ (Roche, Basel, Switzerland) for $6 \mathrm{~h}$ at $37^{\circ} \mathrm{C}[17]$. The meniscal cells were cultured on DMEM containing 10\% FBS and inoculated in 12-well plates. Then, treatment of the cells was conducted with IL-1 $\beta(10 \mathrm{ng} / \mathrm{ml})$ for $2 d$ to achieve the induction of cell senescence injury by inflammatory factors[18]. The meniscus cells were fixed at room temperature for $15 \mathrm{~min}$, after washing three times with PBS, and stained with $\beta$-galactosidase staining solution for $12 \mathrm{~h}$ at $37^{\circ} \mathrm{C}$ without $\mathrm{CO}_{2}$ environment. After that, these were rinsed again with PBS and were observed via an inverted microscope (Nikon, Japan).

\section{Apoptosis assay}

For apoptosis analysis, the meniscus cells treated with IL-1 1 (10ng/ml) for 2 days. After cells resuspended with $100 \mu \mathrm{L} 1 \mathrm{x}$ binding buffer, $5 \mu \mathrm{L}$ Annexin V-FITC and $5 \mu \mathrm{L}$ 7-ADD were added and incubated at room temperature for $15 \mathrm{~min}$ without light. The apoptosis rate was detected by using a FACS Aria III Flow cytometry (BD Biosciences, USA).

\section{Cell immunofluorescence analysis}

For immunofluorescence staining, the meniscus cells were treated with IL-1 $\beta(10 \mathrm{ng} / \mathrm{ml})$ for 2 days, and then the cells were fixed in $4 \%$ paraformaldehyde for 15 minutes at room temperature. After washing with PBS, the cells were permeabilized with $0.3 \%$ Triton X-100 for 15 minutes and blocked with $3 \%$ BSA at room temperature for 40 minutes. Then, the cells were washed with PBS and incubated with primary antibodies of $\operatorname{CDK1}$ (1:200, dilution), $A U R K B$ (1:200, dilution), RRM2 (1:100, dilution), and $T I M P 1$ (1:150, dilution, Abclonal) at $4{ }^{\circ} \mathrm{C}$ overnight. The meniscus cells were rinsed three times with PBS and incubated with secondary antibodies (1:100; ThermoFisher, USA) for $1 \mathrm{~h}$ at room temperature the next day. In the end, we stained the nuclei with 4',6-diamidino-2-phenylindole (DAPI, Thermo Fisher, USA) for 5 minutes. All of the images were captured under the fluorescence microscope (Nikon, Japan).

\section{qRT-PCR confirmation of the hub genes}

Total RNA from meniscus tissue was extracted with TRIzol reagent following the manufacturer's protocol. After the purity and concentration of isolated RNA were determined by the nano-drop-2000 nucleic acid analyzer (ThermoFisher, USA), the RNA reverse transcription kits were devoted to reverse transcribe total 
RNA into cDNA. Quantitative real-time PCR (qRT-PCR) was performed with the ABI StepOnePlus cycler (Applied Biosystems, USA) in a 10- $\mu$ l reaction system. To accurately quantify the gene expression levels, mRNA levels of the internal reference gene glyceraldehyde phosphate dehydrogenase (GAPDH) was normalized by the $2^{-\triangle \triangle C T}$ method. The primer sequences of genes applied in this study were listed in the Table 1.

Table 1

The primer sequences of genes in this experiment

\begin{tabular}{|llll|}
\hline Target genes & Species & Forward primer & Reverse primer \\
\hline$R R M 2$ & homo & GTTTGTGGCAGACAGACTTA & TCACTCCCATCCTCTGATAC \\
\hline$R R M 2$ & mouse & TAGGCGAGTATCAGAGGATG & GTGTAGCCAGTTGGTTGTT \\
\hline AURKB & homo & CCAGAAGGTGATGGAGAATAG & CCCATGGCAGTACATTAGAG \\
AURKB & mouse & CTACAAGGAACTGCAGAAGAG & CAGGTTCTCCGGCTTTATG \\
CDK1 & homo & GTCAGCTCGTTACTCAACTC & CTTCTGGCCACACTTCATTA \\
\hline CDK1 & mouse & CATGGTCAGAGGTAGGTTAGA & CTAAGCAGCACAGCGATAC \\
\hline TIMP1 & homo & CCACCTTATACCAGCGTTATG & CAGGTAGTGATGTGCAAGAG \\
\hline TIMP1 & mouse & GGCATCCTCTTGTTGCTATC & GGTGGTCTCGTTGATTTCTG \\
\hline GAPDH & homo & GAAGGTGAAGGTCGGAGTC & GAAGATGGTGATGGGATTTC \\
\hline $\begin{array}{l}\text { GAPDH } \\
\text { RRM2, ribonucleotide reductase regulatory subunit M2; AURKB, aurora kinase B; CDK1, cyclin } \\
\text { dependent kinase 1; TIMP1, tissue inhibitor of metalloproteinases 1; GAPDH, glyceraldehyde } \\
\text { phosphate dehydrogenase. }\end{array}$ & AGGTCGGTGTGACGGATTG & TGTAGACCATGTAGTTGAGGTCA \\
\hline
\end{tabular}

\section{Statistical analysis}

The expression levels of hub genes that were differentially expressed between young and aging tissues or cells were determined by implementing Student's $t$-test with Prism 6.0 (GraphPad Software, USA), and the data of the experimental results were presented as mean \pm standard error of mean (SEM). In addition, for GO functional annotation, DAVID calculated the $P$ value of the false discovery and enrichment rate via Benjamini correction. The $P<0.05$ was considered to be statistically significant difference.

\section{Results}

\section{Identification of DEGs}

A total of 631 DEGs, including 341 up-regulated genes and 290 down-regulated genes, were identified from the GSE45233 dataset by comparing the gerontic and young meniscus samples. The heatmap and volcano plot of total DEGs were shown in Figure. $1 \mathrm{~A}$ and $\mathrm{B}$, respectively. 


\section{GO function and KEGG pathway enrichment analysis of the DEGs}

The results of the GO analysis showed that DEGs related to $\mathrm{CC}$ were notably concentrated in extracellular region, extracellular space, integral component of plasma membrane, and proteinaceous extracellular matrix (Figure. 2A). Variations in DEGs connected with BP were mainly concentrated in signal transduction, regulation of transcription from RNA polymerase II promoter, and regulation of cell proliferation (Figure. 2B). Regarding MF, DEGs were prominently gathered in calcium ion binding, chromatin binding, sequence-specific DNA binding, and heparin-binding (Figure. 2C). KEGG pathway analysis manifested that the top typical pathways correlated with DEGs were PI3K-Akt signaling pathway, transcriptional misregulation in cancers, and Jak-STAT signaling pathway (Figure. 2D).

\section{Construction of PPI network and hub genes analysis}

The PPI network of the DEGs was constructed by employing STRING (Figure. 3A). The Degree, MCC, MNC, and EPC algorithms were applied to filtrate hub genes shown in Figure. 3B, C, D, and E, respectively. The mutual hub genes (RRM2, AURKB, CDK1, and TIMP1) were discerned by the Venn diagram (Figure. 3F). According to Metascape analysis, $\mathrm{GO}$ enrichment showed that the significant hub genes related to $\mathrm{BP}$ were mainly concentrated in aging and regulation of the cell cycle process (Figure. 3G).

\section{Construction of a IncRNA-miRNA-mRNA network and molecular docking of potential drugs with hub genes}

As for the screening of miRNAs, since there were no miRNAs that could act on all Hub genes, miRNAs working on three Hub genes were selected as ideal targets. Through the miRWalk website predictive analysis, we screened 5 miRNAs (hsa-miR-6810-5p, hsa-miR-4676-5p, hsa-miR-6877-5p, hsa-miR-8085, and hsa-miR-6133) (Figure. 4A, Table 2). As illustrated in Figure. 4B, these miRNAs are referred to some pathways that include glycosaminoglycans biosynthesis. To further understand the function of the hub genes in meniscal degeneration, we employed Cytoscape to combine IncRNA/miRNA interactions with miRNA/mRNA interactions, thus constructing a IncRNA-miRNA-mRNA network (Figure. 4C).

Patients undergoing meniscal resection may have difficulty regaining previous motion abilities.[19, 20] Therefore, preventing meniscus aging is one of the treatment strategies to reduce the incidence of meniscus tearing. Through probing the DGIdb website, we selected the above hub genes for molecular docking analysis in order to ascertain potential drugs that could act on these genes (Figure. 5D). The predicted results showed the high-affinity hydrogen binding events between drugs, including Cladribine, Danusertib, Barasertib, Riviciclib, and Dinaciclib, and residues of hub genes, so it was necessary to consider the possible senescence and degeneration of meniscus caused by these drugs in clinical application. 
Table 2

Information of the 5 miRNAs most relevant to the common hub genes.

\begin{tabular}{|c|c|c|c|c|c|}
\hline & Target genes & Position & Energy & $\begin{array}{l}\text { Binding region } \\
\text { length }\end{array}$ & P-value \\
\hline $\begin{array}{l}\text { hsa-miR-6810- } \\
5 p\end{array}$ & RRM2,CDK1,TIMP1 & 3UTR & -24.1 & 29 & 0.01664477 \\
\hline $\begin{array}{l}\text { hsa-miR-4676- } \\
5 p\end{array}$ & RRM2,CDK1,TIMP1 & 3UTR & -22.9 & 18 & 0.007139029 \\
\hline $\begin{array}{l}\text { hsa-miR-6877- } \\
5 p\end{array}$ & RRM2,CDK1,TIMP1 & 3UTR & -21.8 & 24 & 0.000873123 \\
\hline hsa-miR-8085 & RRM2,CDK1,TIMP1 & 3UTR & -22.6 & 25 & 0.006883728 \\
\hline hsa-miR-6133 & RRM2,AURKB,TIMP1 & 3UTR & -22.4 & 17 & 0.001472693 \\
\hline
\end{tabular}

RRM2, ribonucleotide reductase regulatory subunit M2; AURKB, aurora kinase B; CDK1, cyclin dependent kinase 1 ; TIMP1, tissue inhibitor of metalloproteinases 1.

\section{The expression of AURKB, RRM2, CDK1, and TIMP1 were decreased in senescent meniscus tissues}

Safranin 0-Fast green and Toloniumchloride Blue staining results of the meniscus of 18-month-old aging mice revealed more severe degenerative histopathological changes than the meniscus in 3-month-old young mice due to decreased collagen fiber content (Figure.5A). Immunohistochemistry showed that the expression levels of $A U R K B, R R M 2, C D K 1$, and TIMP1 protein were higher in the meniscus of young mice than that of the senescent mice (Figure.5B and $C$ ). In order to further explore the expression levels of hub genes in senescent meniscus, we applied human meniscus for immunohistochemical detection. The results illustrated that the Hub genes were also poorly expressed in senescent human meniscus (Figure.5D and E). Moreover, the mRNA levels of $A U R K B, R R M 2, C D K 1$, and TIMP1 were also decreased both in human and mouse menisci, respectively (Figure.5F). These founds suggested that the decreased gene expressions of $A U R K B, R R M 2, C D K 1$, and TIMP1 might be a trigger or indicator that participating in meniscus aging and degeneration.

\section{Hub genes expression in cultured mouse meniscus cells}

As an additional method to further investigate the expressions of hub genes in senescent meniscus tissues, we treated normal mouse meniscus cells with IL-1 $13(10 \mathrm{ng} / \mathrm{ml})$ for $48 \mathrm{~h}$ to induce degeneration in mouse meniscus cells in vitro. Take into consideration that the overexpression and accumulation of endogenous lysosomal $\beta$-galactosidase are one of the characteristics of senile cells, SA- $\beta$-gal is often taken as a biomarkers of senile cells.[21, 22] More positive stained cells, the blue ones, were observed in the IL-1 $\beta$ group than the control by the SA- $\beta$-gal staining (Figure. $6 \mathrm{~A}$ ). Meanwhile, more apoptotic cells were detected in the IL-1 $\beta$ group than the control by flow cytometry (Figure. 6B and D). In addition, the suppressed mRNAs expressions of $A U R K B, R R M 2, C D K 1$, and TIMP1 were detected in meniscus cells treated with IL-1 $\beta(10 \mathrm{ng} / \mathrm{ml})$ when compared with the control group (Figure. $6 \mathrm{C})$. Then, the immunofluorescence assay further supported that these Hub genes were poor expressions in senescent 
meniscus cells (Figure. 6E). These consequences further confirmed the decreased AURKB, RRM2, CDK1, and TIMP1 expressions in the senescent meniscus.

\section{Discussion}

The incidence of meniscal injuries is on the rise, which might be partly attributed to the general aging of the population[23]. Previous studies have found that age-related meniscus denaturation induces an increase in fragility of meniscus tissue, thereby increasing the incidence of meniscus tearing in the elderly population[24]. However, the molecular pathologic mechanism of meniscus age-related degeneration is still poorly studied[25]. The high-throughput microarray technology combined with bioinformatics analysis has been widely used to predict the pathogenesis and potential molecular therapeutic targets of many diseases.

In the current study, a microarray dataset GSE45233 containing young and gerontic meniscus samples was obtained and a bioinformatics analysis was completed. In view of the results of GO terms enrichment analysis, we found a relationship between the DEGs and cell senescence. According to the CC analysis results of DEGs, we found that the major changes in the components of the cells were located in the extracellular region. The extracellular domain of meniscus cells mainly refers to the extracellular matrix $(E C M)$, which plays a crucial role in sustaining structural integrity and mechanical properties of meniscus[26]. Histopathological analysis showed that meniscus aging was associated with ECM structure loss[27]. Variations in DEGs related to MF were primarily concentrated in calcium ion binding, and chromatin binding. In fact, imbalance of calcium ion homeostasis and chromatin rearrangement are also often associated with cell senescence and ECM degeneration[28, 29]. Based on the KEGG database, we discovered that the DEGs were principally gathered in the PI3K-Akt signaling pathway, which could act on downstream targets including forkhead box 0 transcription factors (FoxO) and mammalian target of rapamycin ( $m T O R)$, and also activate gene $P 53$ to regulate cell growth[30-32]. Herranz et al. found that $m T O R$ controls senescence secretory by indirectly regulating the expressions of senescence signaling molecules[33]. Furthermore, FoxO may play a protective role against meniscus aging by regulating autophagy[34]. Such reports further confirmed our finding.

The PPI network was carried out to predict the connections of protein functions of DEGs, and the common hub genes- $A U R K B, C D K 1, R R M 2$, and TIMP1-were screened. CDK1 is a catalytic subunit of mitogenic promotors and plays a key role in cell cycle control, such as mitosis and G2-M transformation[35]. Saito et al. demonstrated that $C D K 1$ was essential for chondrocyte proliferation and differentiation[36]. AURKB is a type of Aurora kinase involved in the regulation of chromosome alignment and separation in mitosis and meiosis[37]. Thus, both AURKB and CDK1 could reflect the meniscus cell cycle to a certain degree. RRM2 encodes one of two non-identical subunits for ribonucleotide reductase, and catalyzes the formation of deoxyribonucleotides from ribonucleotides[38]. Previous studies have found that the decrease in $R R M 2$ levels could lead to DNA damage accumulation by inhibiting deoxyribonucleotide triphosphates (dNTPs) expression, thereby mediating cell senescence[39]. TIMP1, natural inhibitors of the matrix metalloproteinases (MMPs), is a group of peptidases involved in 
degradation of the extracellular matrix[40]. The balance between matrix degradation and synthesis is impaired during meniscus aging. Since elastolysis or collagenolysis is put down to the joint effect of several members of MMPs, TIMP1 may also play an anti-meniscus aging role to some extent[41]. In the current study, the decrease in the expressions of $A U R K B, C D K 1, R R M 2$, and TIMP1 was observed in both the human and mouse senescent menisci, as well as meniscus cells treated with exogenous IL-1 $\beta$. Such finding indicated that both cell cycle arrest and ECM degeneration contribute to the meniscus senescence.

For the five selected key miRNAs targeting the hub genes, there were no relevant studies reporting that they were linked to meniscus before. Further pathways enrichment analysis of these miRNA revealed that these miRNAs were involved in the synthesis of glycosaminoglycans, an important component of the extracellular matrix of the meniscus, of which, the reduction was often accompanied by the occurrence of OA[42]. With regard to the drugs (Cladribine, Danusertib, Barasertib, Riviciclib, and Dinaciclib) targeting hub genes based on pharmacogenomics tools prediction, their toxic and side effects on meniscus aging and damage should also be taken into account in the treatment of related diseases[43]. Cellular senescence is a complex phenotype characterized by two aspects: persistent cell cycle arrest and the production of pro-inflammatory molecules known as senescence associated secretion phenotype (SASP) [44]. Considering that the senescence of articular chondrocytes acting as hyporeplicative cells was mainly driven by SASP factors, we utilized IL-1 $\beta(10 \mathrm{ng} / \mathrm{ml})$ to construct an in vitro cell senescence model[45]. Our current study has shown that cell senescence was increased in IL-1 $\beta$-treated mouse meniscus cells. The decreased expressions of screened hub genes in vitro meniscus cells supported in vivo experiments and further identified these genes were related to cell senescence.

However, our conclusions are only originated from theoretical prediction and models detection, and the role of pivotal genes in meniscus senescence still needs further clinical verification. Still, our current study would provide a basis for finding markers of the aging meniscus to a certain extent.

\section{Declarations}

\section{Ethics approval and consent to participate}

This human research protocol was authorized by the Medical Ethics Committees of Renmin Hospital of Wuhan University (approval number: 2019K-K011). All patients have signed informed consent. Animal protocols used in this study were approved by the Animal Welfare Committee in Wuhan University (License number: 14016). These approvals included consents to the collection, storage, and testing of samples for research conducted in this manuscript.

\section{Consent for publication}

Not Applicable

\section{Availability of data and materials}


The protein sequences and structures from this study were deposited in RCSB PDB database and were accessible through PDB ticket number 6C2R, 2B9R, and 6LE1,

at: https://www.rcsb.org/structure/6C2R, https://www.rcsb.org/structure/2B9R, and https://www.rcsb.org/structure/6LE1.

The DNA and RNA sequencing data were downloaded from Genebank database through gene names RRM2 (Gene ID: 6241 and 20135), CDK1(Gene ID: 983 and 12534), AURKB (Gene ID: 9212 and 20877), and TIMP1(Gene ID: 7076 and 21857). Then, the microarray data used in this study could be obtained through GEO ticket number GSE45233 in the GEO database (https://www.ncbi.nlm.nih.gov/gds/? term=GSE45233/).

Finally, the crystallographic data for drugs of this study were stored in the PubChem database and could be searchable by drug compound CID 20279

(Cladribine, https://pubchem.ncbi.nlm.nih.gov/compound/20279), 11442891

(Danusertib, https://pubchem.ncbi.nlm.nih.gov/compound/11442891), 11497983

(Barasertib, https://pubchem.ncbi.nlm.nih.gov/compound/11497983), 23643976

(Riviciclib, https://pubchem.ncbi.nlm.nih.gov/compound/23643976), and 46926350 (Dinaciclib, https://pubchem.ncbi.nlm.nih.gov/compound/46926350).

\section{Competing interests}

No competed or conflicts of interest reported.

\section{Funding}

This work was supported by the National Natural Science Foundation of China (No. 81673490, 81603214, 81972036), the National Key Research and Development Project of China (No. 2020YFA0803900), and the Key Research and Development Project of Hubei province (No. 2020BCA071). No benefits in any form have been received or will be received from a commercial party related directly or indirectly to the subject of this article.

\section{Authors' contributions}

Chen L.B., Wen Y.X., and Chen M. designed the research study; Chen M. and Zhou SQ. performed the research; Chen M., Zhou SQ., Shi HS., and Gu HW. analyzed the data; Chen M., Zhou SQ., Chen L.B., and Wen Y.X. wrote and revised the paper. All authors read and approved the final manuscript.

\section{Acknowledgements}

Not Applicable.

\section{References}


1. Liu $\mathrm{F}, \mathrm{Xu} \mathrm{H}$, Huang $\mathrm{H}$. A novel kartogenin-platelet-rich plasma gel enhances chondrogenesis of bone marrow mesenchymal stem cells in vitro and promotes wounded meniscus healing in vivo. Stem cell research \& therapy. 2019; 10(1):201.

2. Bhan K. Meniscal Tears: Current Understanding, Diagnosis, and Management. Cureus. 2020; 12(6):e8590.

3. Mitchell J, Graham W, Best TM, Collins C, Currie DW, Comstock RD, Flanigan DC. Epidemiology of meniscal injuries in US high school athletes between 2007 and 2013. Knee surgery, sports traumatology, arthroscopy: official journal of the ESSKA. 2016; 24(3):715-722.

4. Persson F, Turkiewicz A, Bergkvist D, Neuman P, Englund M. The risk of symptomatic knee osteoarthritis after arthroscopic meniscus repair vs partial meniscectomy vs the general population. Osteoarthritis and cartilage. 2018; 26(2):195-201.

5. Li L, Yang X, Yang L, Zhang K, Shi J, Zhu L, Liang H, Wang X, et al. Biomechanical analysis of the effect of medial meniscus degenerative and traumatic lesions on the knee joint. American journal of translational research. 2019; 11(2):542-556.

6. Özdemir M, Kavak R. Meniscal Lesions in Geriatric Population: Prevalence and Association with Knee Osteoarthritis. Current aging science. 2019; 12(1):67-73.

7. Wu J, Huang JM, Zhao B, Cao JG, Chen X. Risk Factors Comparison for Radial and Horizontal Tears. The journal of knee surgery. 2016; 29(8):679-683.

8. Tsujii A, Nakamura N, Horibe S. Age-related changes in the knee meniscus. The Knee. 2017; 24(6):1262-1270.

9. Kwok J, Onuma H, Olmer M, Lotz MK, Grogan SP, D'Lima DD. Histopathological analyses of murine menisci: implications for joint aging and osteoarthritis. Osteoarthritis and cartilage. 2016; 24(4):709-718.

10. Sarbacher CA, Halper JT. Connective Tissue and Age-Related Diseases. Sub-cellular biochemistry. 2019; 91:281-310.

11. Rodier F, Campisi J. Four faces of cellular senescence. The Journal of cell biology. 2011; 192(4):547-556.

12. Brophy RH, Sandell LJ, Rai MF. Traumatic and Degenerative Meniscus Tears Have Different Gene Expression Signatures. The American journal of sports medicine. 2017; 45(1):114-120.

13. Liu L, Song B, Ma J, Song Y, Zhang SY, Tang Y, Wu X, Wei Z, et al. Bioinformatics approaches for deciphering the epitranscriptome: Recent progress and emerging topics. Computational and structural biotechnology journal. 2020; 18:1587-1604.

14. Rai MF, Patra D, Sandell LJ, Brophy RH. Transcriptome analysis of injured human meniscus reveals a distinct phenotype of meniscus degeneration with aging. Arthritis and rheumatism. 2013; 65(8):2090-2101.

15. Huang P, Gu J, Wu J, Geng L, Hong Y, Wang S, Wang M. Microarray analysis of the molecular mechanisms associated with age and body mass index in human meniscal injury. Molecular medicine reports. 2019; 19(1):93-102. 
16. Ding J, Li X, Hu H. TarPmiR: a new approach for microRNA target site prediction. Bioinformatics (Oxford, England). 2016; 32(18):2768-2775.

17. Fu XN, Li HW, Du N, Liang X, He SH, Guo KJ, Li TF. Erythropoietin enhances meniscal regeneration and prevents osteoarthritis formation in mice. American journal of translational research. 2020; 12(10):6464-6477.

18. Diekman BO, Sessions GA, Collins JA, Knecht AK, Strum SL, Mitin NK, Carlson CS, Loeser RF, et al. Expression of p16(INK) (4a) is a biomarker of chondrocyte aging but does not cause osteoarthritis. Aging cell. 2018; 17(4):e12771.

19. Sood M, Kulshrestha V, Sachdeva J, Ghai A, Sud A, Singh S. Poor Functional Outcome in Patients with Voluntary Knee Instability after Anterior Cruciate Ligament Reconstruction. Clinics in orthopedic surgery. 2020; 12(3):312-317.

20. Zhang K, Li L, Yang L, Shi J, Zhu L, Liang H, Wang X, Yang X, et al. Effect of degenerative and radial tears of the meniscus and resultant meniscectomy on the knee joint: a finite element analysis. Journal of orthopaedic translation. 2019; 18:20-31.

21. Ke Y, Li D, Zhao M, Liu C, Liu J, Zeng A, Shi X, Cheng S, et al. Gut flora-dependent metabolite Trimethylamine-N-oxide accelerates endothelial cell senescence and vascular aging through oxidative stress. Free radical biology \& medicine. 2018; 116:88-100.

22. Singh M, Piekorz RP. Senescence-associated lysosomal a-L-fucosidase (SA-a-Fuc): a sensitive and more robust biomarker for cellular senescence beyond SA- $\beta$-Gal. Cell cycle (Georgetown, Tex). 2013; 12(13):1996.

23. Chambers HG, Chambers RC. The Natural History of Meniscus Tears. Journal of pediatric orthopedics. 2019; 39(Issue 6, Supplement 1 Suppl 1):S53-s55.

24. Wesdorp MA, Eijgenraam SM, Meuffels DE, Bierma-Zeinstra SMA, Kleinrensink GJ, BastiaansenJenniskens YM, Reijman M. Traumatic Meniscal Tears Are Associated With Meniscal Degeneration. The American journal of sports medicine. 2020; 48(10):2345-2352.

25. Di Giancamillo A, Deponti D, Modina S, Tessaro I, Domeneghini C, Peretti GM. Age-related modulation of angiogenesis-regulating factors in the swine meniscus. Journal of cellular and molecular medicine. 2017; 21(11):3066-3075.

26. López-Franco M, Gómez-Barrena E. Cellular and molecular meniscal changes in the degenerative knee: a review. Journal of experimental orthopaedics. 2018; 5(1):11.

27. Pauli C, Grogan SP, Patil S, Otsuki S, Hasegawa A, Koziol J, Lotz MK, D'Lima DD. Macroscopic and histopathologic analysis of human knee menisci in aging and osteoarthritis. Osteoarthritis and cartilage. 2011; 19(9):1132-1141.

28. Adams PD. Remodeling chromatin for senescence. Aging cell. 2007; 6(4):425-427.

29. Sun Y, Mauerhan DR. Meniscal calcification, pathogenesis and implications. Current opinion in rheumatology. 2012; 24(2):152-157.

30. Kastenhuber ER, Lowe SW. Putting p53 in Context. Cell. 2017; 170(6):1062-1078. 
31. Zhang X, Tang N, Hadden TJ, Rishi AK. Akt, FoxO and regulation of apoptosis. Biochimica et biophysica acta. 2011; 1813(11):1978-1986.

32. Karar J, Maity A. PI3K/AKT/mTOR Pathway in Angiogenesis. Frontiers in molecular neuroscience. 2011; 4:51.

33. Herranz N, Gallage S, Mellone M, Wuestefeld T, Klotz S, Hanley CJ, Raguz S, Acosta JC, et al. mTOR regulates MAPKAPK2 translation to control the senescence-associated secretory phenotype. Nature cell biology. 2015; 17(9):1205-1217.

34. Lee KI, Choi S, Matsuzaki T, Alvarez-Garcia O, Olmer M, Grogan SP, D'Lima DD, Lotz MK. FOXO1 and FOXO3 transcription factors have unique functions in meniscus development and homeostasis during aging and osteoarthritis. Proceedings of the National Academy of Sciences of the United States of America. 2020; 117(6):3135-3143.

35. Santamaría D, Barrière C, Cerqueira A, Hunt S, Tardy C, Newton K, Cáceres JF, Dubus P, et al. Cdk1 is sufficient to drive the mammalian cell cycle. Nature. 2007; 448(7155):811-815.

36. Saito M, Mulati M, Talib SZ, Kaldis P, Takeda S, Okawa A, Inose H. The Indispensable Role of CyclinDependent Kinase 1 in Skeletal Development. Scientific reports. 2016; 6:20622.

37. Ma HT, Poon RYC. Aurora kinases and DNA damage response. Mutation research. 2020; 821:111716.

38. Coelho MB, Ascher DB, Gooding C, Lang E, Maude H, Turner D, Llorian M, Pires DE, et al. Functional interactions between polypyrimidine tract binding protein and PRI peptide ligand containing proteins. Biochemical Society transactions. 2016; 44(4):1058-1065.

39. Aird KM, Zhang R. Metabolic alterations accompanying oncogene-induced senescence. Molecular \& cellular oncology. 2014; 1(3):e963481.

40. Zhu Z, Zhong L, Li R, Liu Y, Chen X, Li Z, Huang L. Study of Osteoarthritis-Related Hub Genes Based on Bioinformatics Analysis. BioMed research international. 2020; 2020:2379280.

41. Hornebeck W. Down-regulation of tissue inhibitor of matrix metalloprotease-1 (TIMP-1) in aged human skin contributes to matrix degradation and impaired cell growth and survival. Pathologiebiologie. 2003; 51(10):569-573.

42. Wang FS, Kuo CW, Ko JY, Chen YS, Wang SY, Ke HJ, Kuo PC, Lee CH, et al. Irisin Mitigates Oxidative Stress, Chondrocyte Dysfunction and Osteoarthritis Development through Regulating Mitochondrial Integrity and Autophagy. Antioxidants (Basel, Switzerland). 2020; 9(9).

43. Rolfes L, Pfeuffer S, Hackert J, Pawlitzki M, Ruck T, Sondermann W, Korsen M, Wiendl H, et al. Skin Reactions in Patients With Multiple Sclerosis Receiving Cladribine Treatment. Neurology(R) neuroimmunology \& neuroinflammation. 2021; 8(3).

44. Childs BG, Durik M, Baker DJ, van Deursen JM. Cellular senescence in aging and age-related disease: from mechanisms to therapy. Nature medicine. 2015; 21(12):1424-1435.

45. Jeon OH, Kim C, Laberge RM, Demaria M, Rathod S, Vasserot AP, Chung JW, Kim DH, et al. Local clearance of senescent cells attenuates the development of post-traumatic osteoarthritis and creates a pro-regenerative environment. Nature medicine. 2017; 23(6):775-781. 
Figures
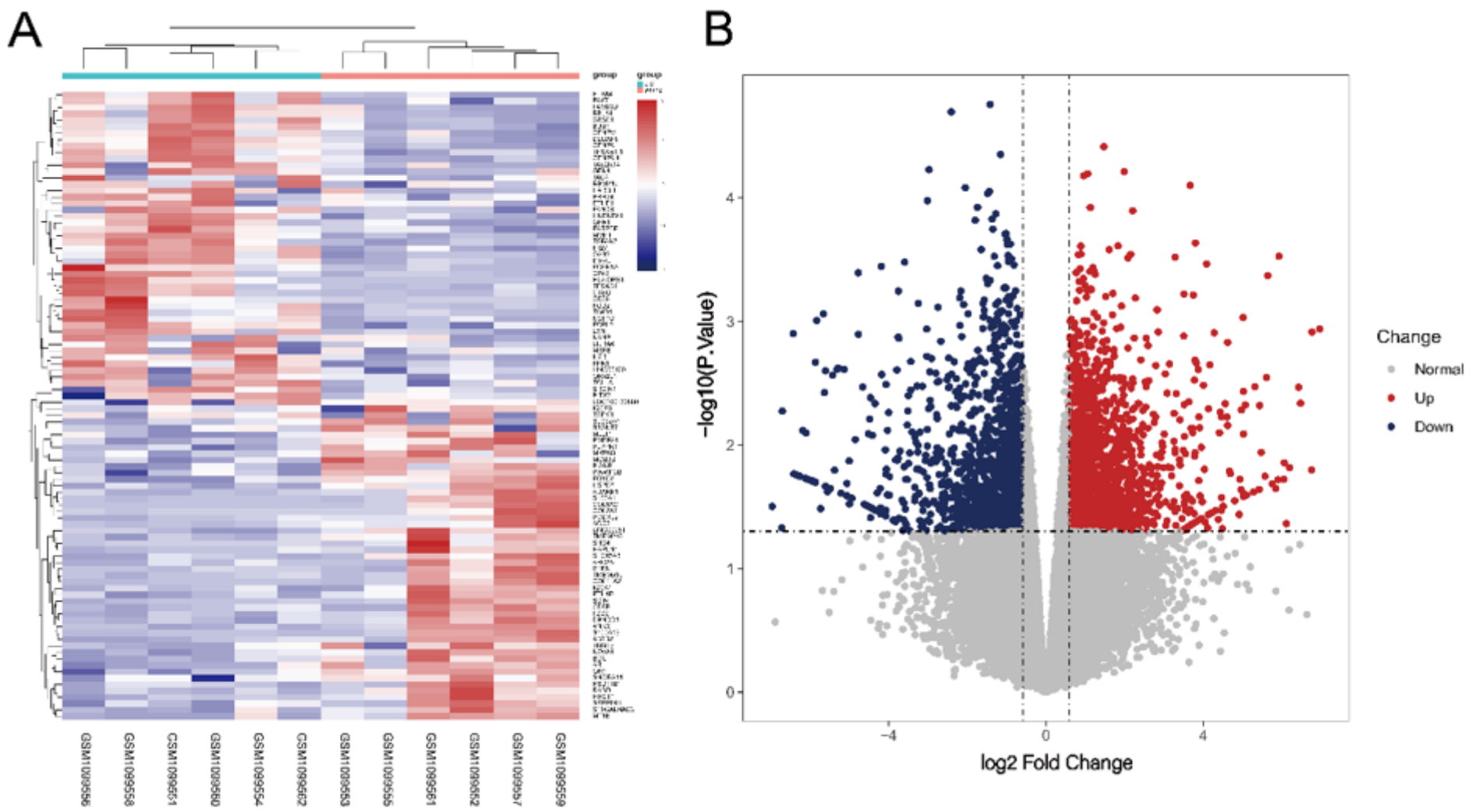

Figure 1

The heatmap and volcano plot of differentially expressed genes (DEGs) between gerontic and younger groups. (A) DEGs expression heatmap of meniscus tissues. (B) DEGs was screened by volcano map filtering method (P-value $<0.05$ and $|\log 2 F C|>1.5)$. "log2FC" means Log2 of the fold change. 
A

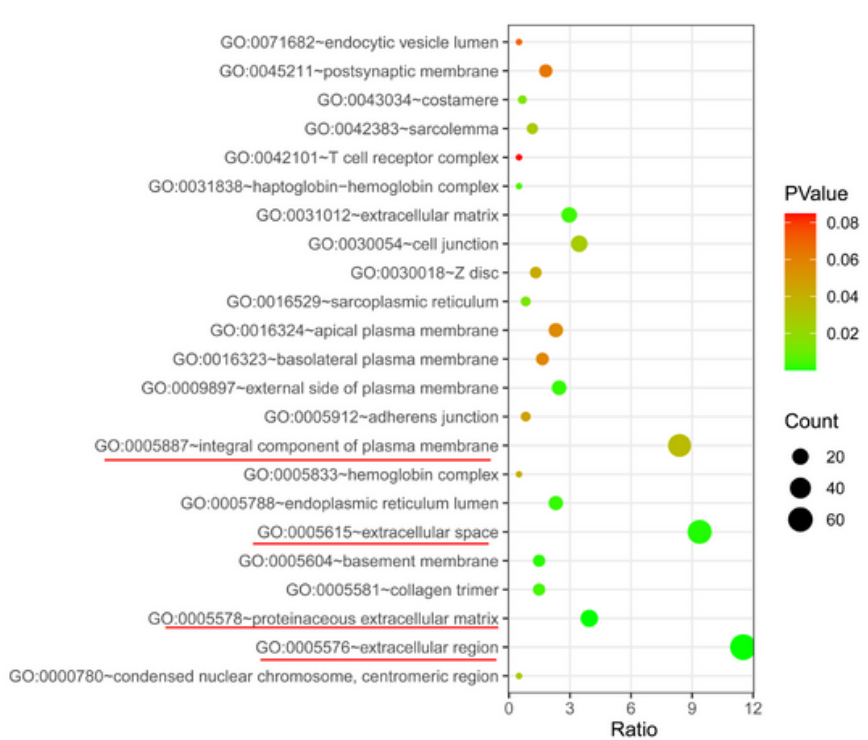

C

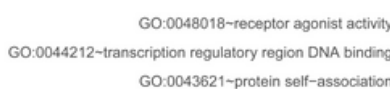
60:0043565-sequence-spocific DNA binding GO:0031720-haptoglobin binding G0:0030246-carbohydrate binding 60:0030020 -extracellular matrix structural constituent conferring tensile strength GO:0008236 -serine-type peptidase activity G0:0008201 heparin binding GO:0008191 metalloendopeptidase inhibitor activity - GO:0005520-insulin-like growh factor binding - GO:0005509 calcium ion binding 60:0005507 copper ion binding G0:0005506-iron ion binding GO:0005344 - oxygen transporter activity GO:0005201 extracellular matrix structural constituent GO:0004896 -cytokine receptor activity Go:0004867-serine-type endopeptidase inhibitor activity 60:0003810-protein-glutamine gamma-glutamyltransferase activity - • GO:0003682-chromatin binding GO:0001786-phosphatidylserine binding

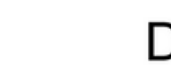

D
$B$

GO-0045944 - Go.0050900 - leukocyle migration Go:0050900-leukocyte migration. $\frac{\text { GO:0045944 regulation of transcription from RNA polymerase II promoter }}{G 0: 0043524 \sim \text { negative regulation of neuron apoptotic process }}$ G0:0043066-negative regulation of apoptotic process GO:0032496-response to lipopolysaccharide GO:0030574-collagen catabolic process GO:0030198-extracellular matrix organization GO:0010951 negative regulation of endopeptidase activity GO:0010628 - positive regulation of gene expression GO:0008285 negative regulation of cell proliferation GO:0008284 positive regulation of cell proliferation GO:0008283-cell proliferation GO:0007568-aging GO:0007283 -spermatogenesis GO:0007165-signal transduction GO:0006955-immune response GO:0006935-chemotaxis GO:0001501-skeletal system development G0:0000122 -negative regulation of transcription from RNA polymerase II
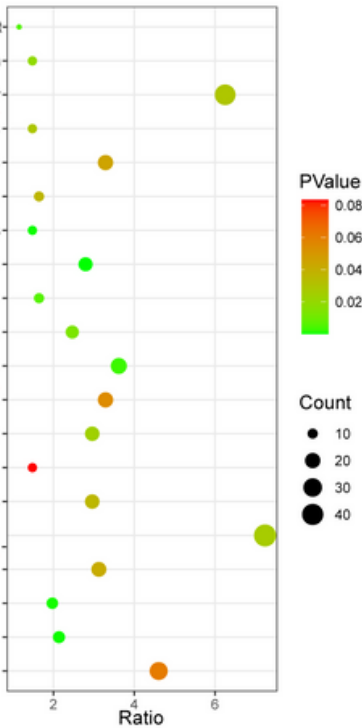

\section{Count}

- 10

20

30
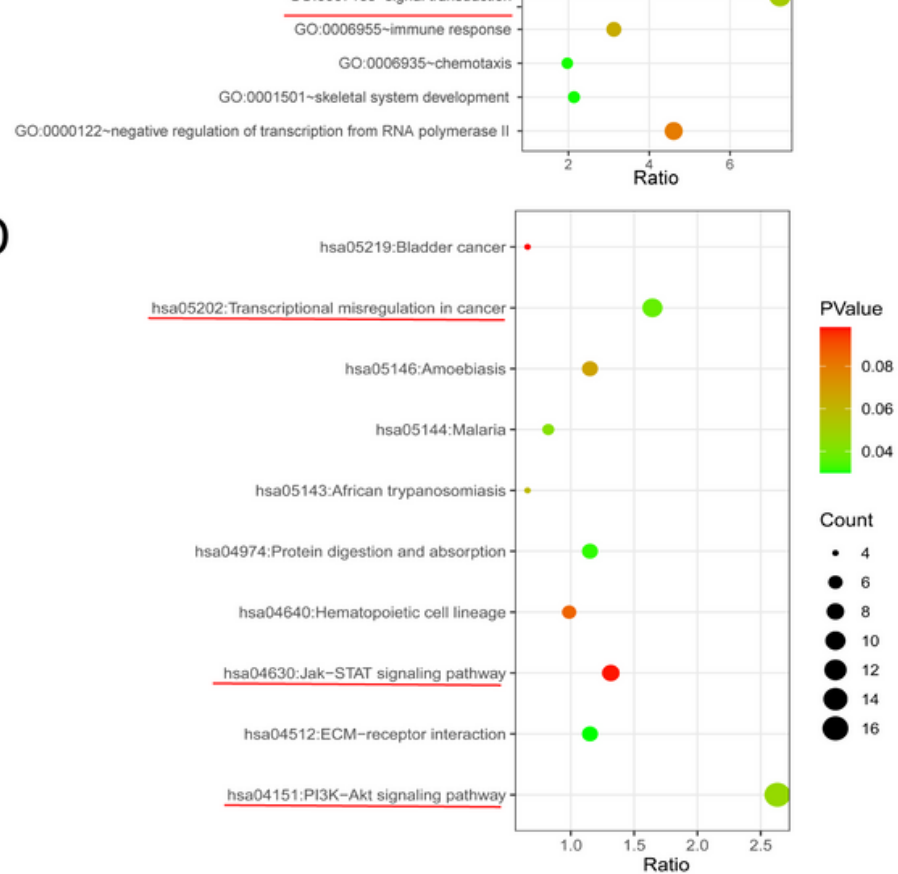

Figure 2

The functional enrichment analysis bubble diagrams of differentially expressed genes (DEGs) by DAVID. Detailed variations information bubble diagrams of GO categories: (A) CC, (B) BP, and (C) MF. (D) KEGG pathway analysis bubble diagram for DEGs. GO, Gene Ontology; CC, cellular component; BP, biological processes; MF, molecular functions; KEGG, Kyoto Encyclopedia of Genes and Genomes. 

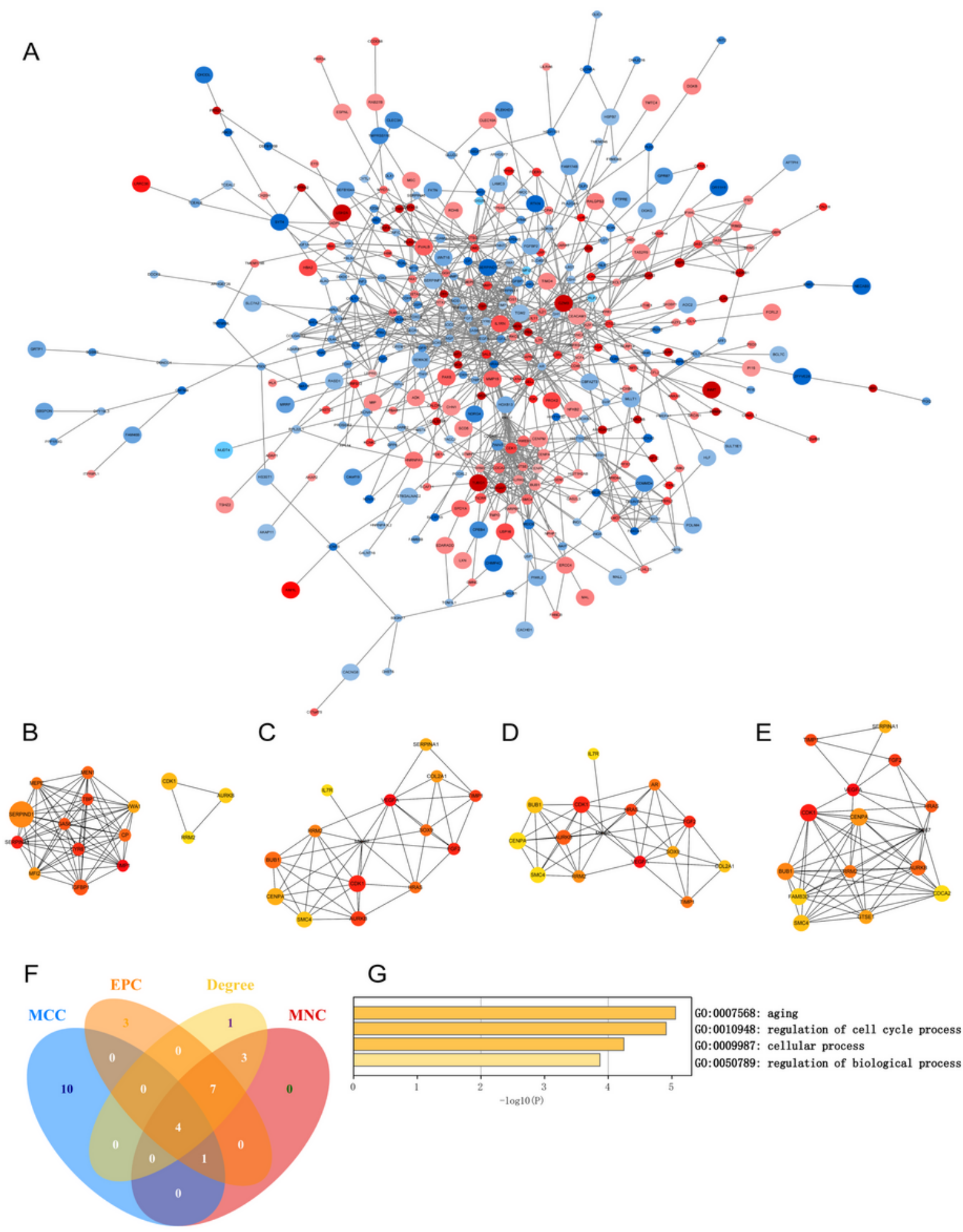

\section{Figure 3}

The PPI network and Hub genes analysis. (A) PPI network of all DEGs $₫ T$ The red codes represent upregulated genes in the elderly, the blue codes represent downregulated genes in the elderly, and the size of the nodes represents the correlation between them). Hub gene networks obtained from the PPI network relying on (B) the Maximal Clique Centrality (MCC) algorithm, (C) the Maximum Neighborhood Component (MNC) algorithm, (D) the Degree algorithm, and (E) the Edge Percolated Component (EPC) 
algorithm of the Cytoscape plugin cytoHubba. (F) The Venn diagram showed the identified hub genes (RRM2, AURKB, CDK1, and TIMP1). (G) The heatmap of common hub genes identified by Metascape was colored by - $\log 10$ (P-value). PPI, protein-protein interaction; DEGs, differentially expressed genes; RRM2, ribonucleotide reductase regulatory subunit M2; AURKB, aurora kinase B; CDK1, cyclin dependent kinase 1; TIMP1, tissue inhibitor of metalloproteinases 1.

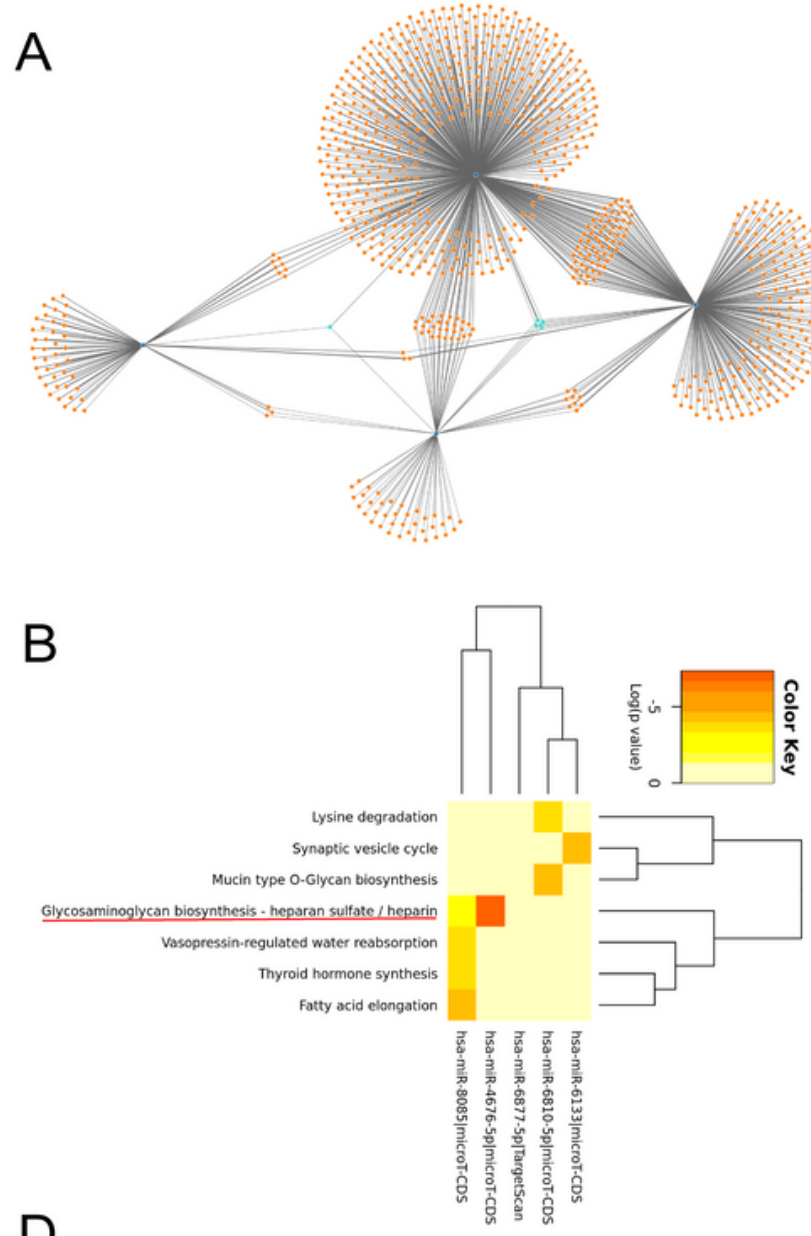

\section{C}
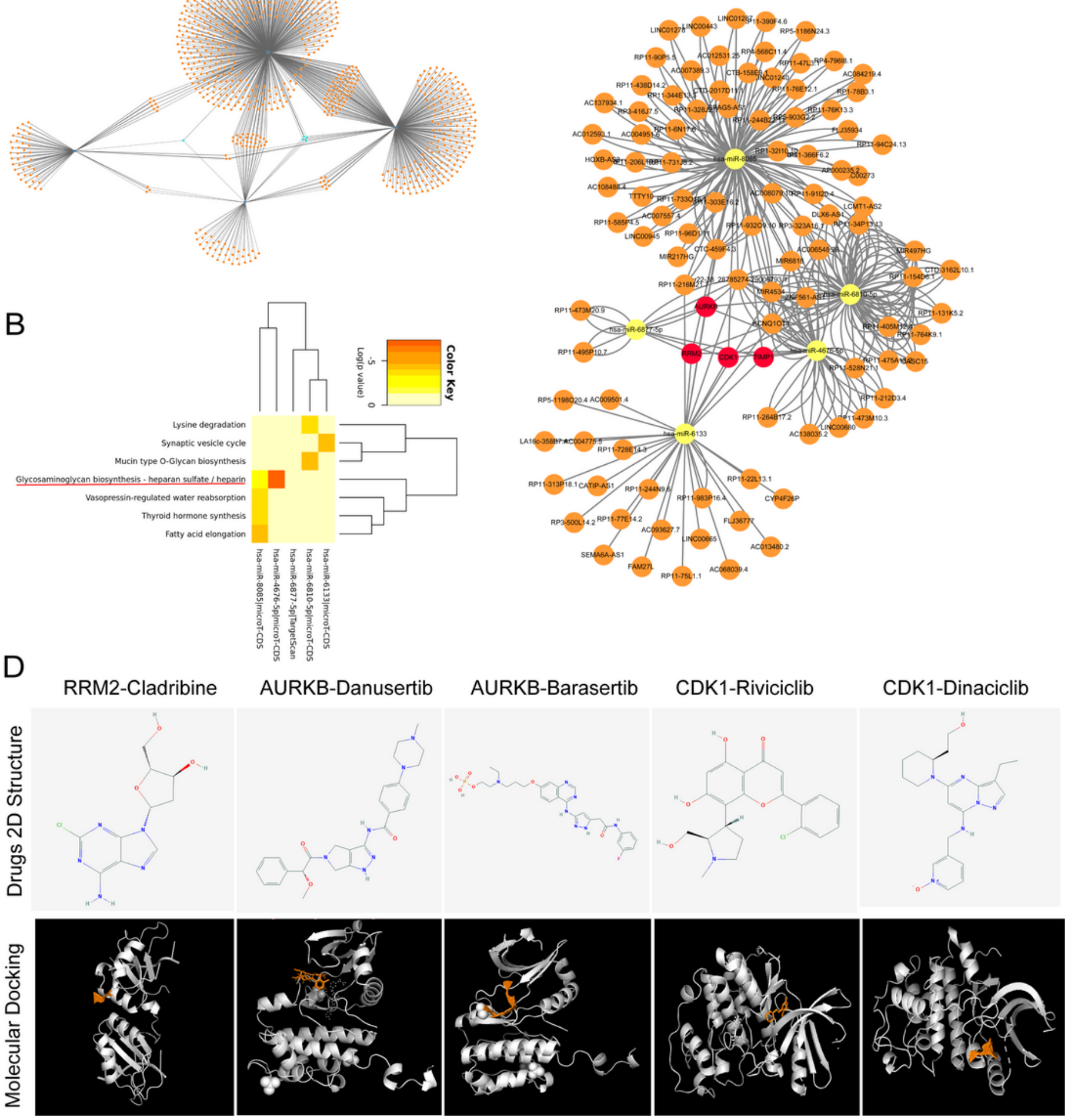

Figure 4 
Network construction of IncRNA-miRNA-mRNA and molecular docking of potential drugs with hub genes. (A) The mRNA-miRNA network was constructed by using the miRWalk website (The five miRNAs most associated with the common hub gene are highlighted in light blue). (B) Meaningful signal pathways about the 5 miRNAs by utilizing the DIANA-miRPath. (C)The IncRNA-miRNA-mRNA regulatory network (The orange nodes represent the IncRNAs, the yellow nodes represent key miRNAs, and the red nodes represent the hub genes). (D) The molecular docking between potential drugs and hub genes (The white structures represent the gene protein residues, and the orange structures represent the 3D models of predicting drugs). RRM2, ribonucleotide reductase regulatory subunit M2; AURKB, aurora kinase B; CDK1, cyclin dependent kinase 1; TIMP1, tissue inhibitor of metalloproteinases 1. 


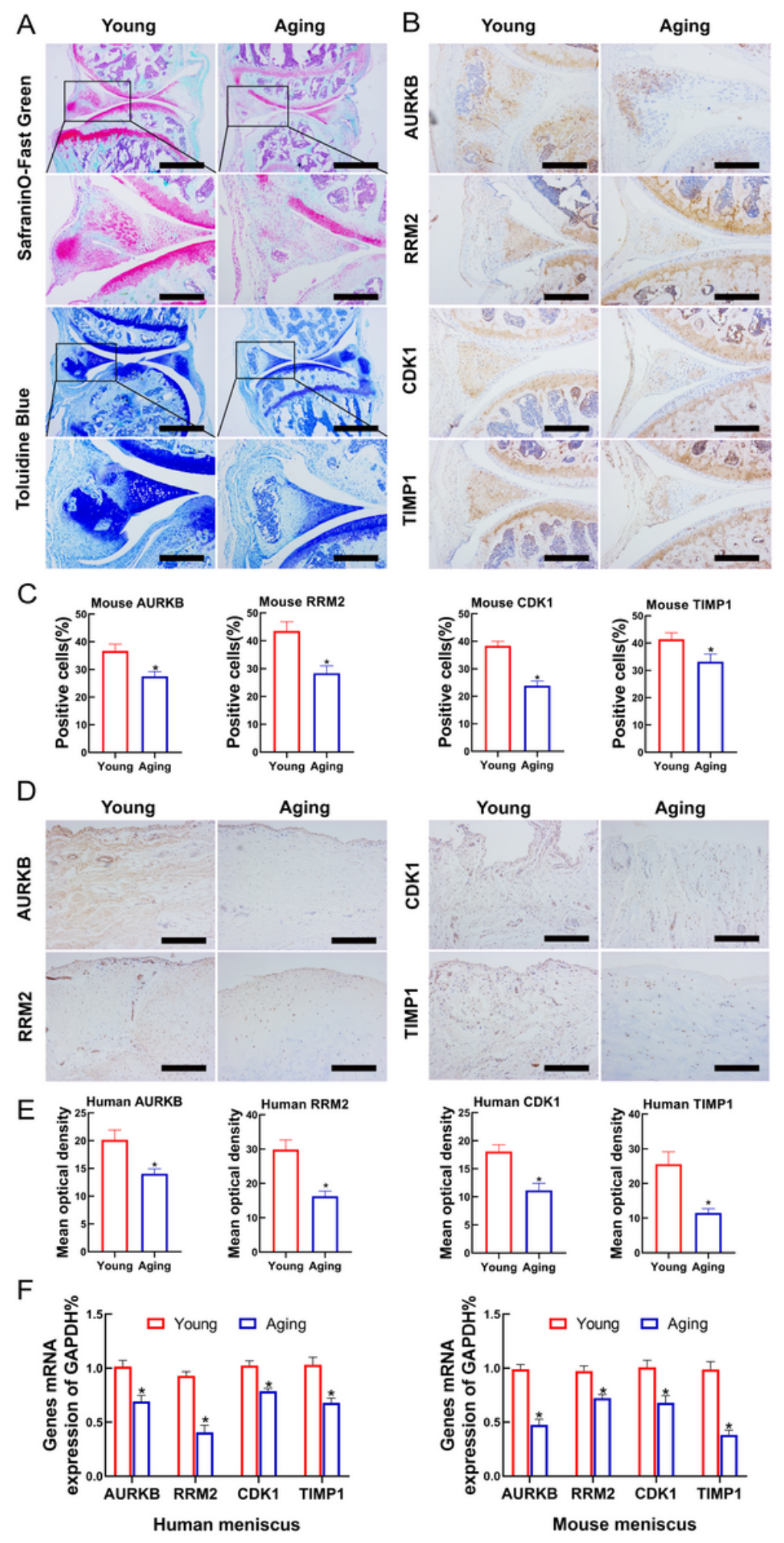

\section{Figure 5}

The expression levels of Hub genes in the meniscus tissues. (A) SafraninO-Fast green and Toloniumchloride Blue staining results of meniscus in 18-month-old mice (aging) and 3-month-old mice (young). Scale bar: $250 \mu \mathrm{m}$. Scale bar (enlarged): $100 \mu \mathrm{m}$. (B) Hub genes immunochemical staining of meniscus in young mice and older mice. Scale bar: $100 \mu \mathrm{m}$. (C) Quantification of Hub genes immunostaining (mean optical density). (D) Immunostaining of Hub genes in young and gerontic human 
menisci. Scale bar: $100 \mu \mathrm{m}$. (E) Quantification of Hub genes immunostaining (mean optical density). (F) The mRNA expression levels of Hub genes in human and mouse menisci, respectively. We normalized the gene expression levels to GAPDH. RRM2, ribonucleotide reductase regulatory subunit M2; AURKB, aurora kinase B; CDK1, cyclin dependent kinase 1; TIMP1, tissue inhibitor of metalloproteinases 1; GAPDH, Glyceraldehyde 3-phosphate dehydrogenase. $n=6$ per group, mean \pm S.E.M., ${ }^{*} P<0.05$ compared with the young group.

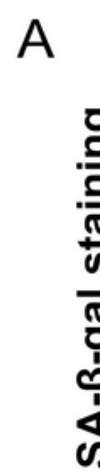

B

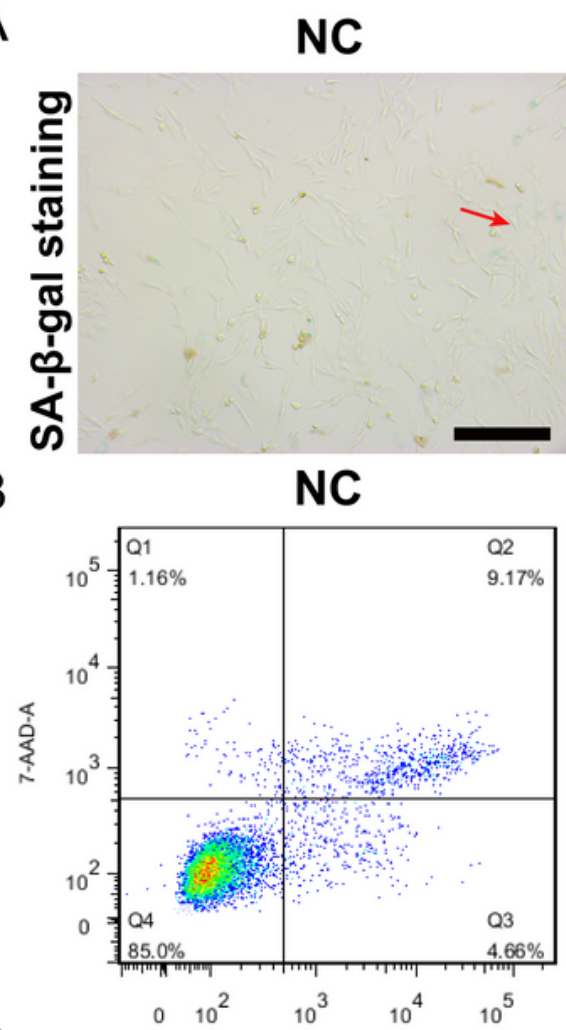

$\mathrm{E}$
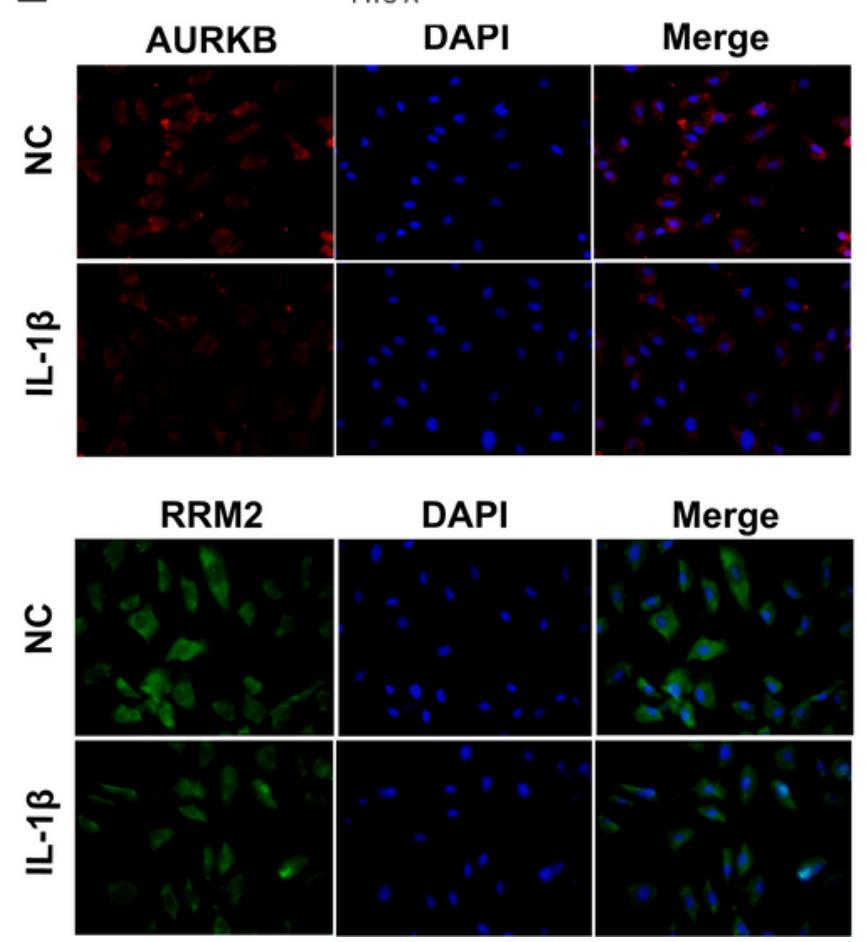

IL-1 $\beta$

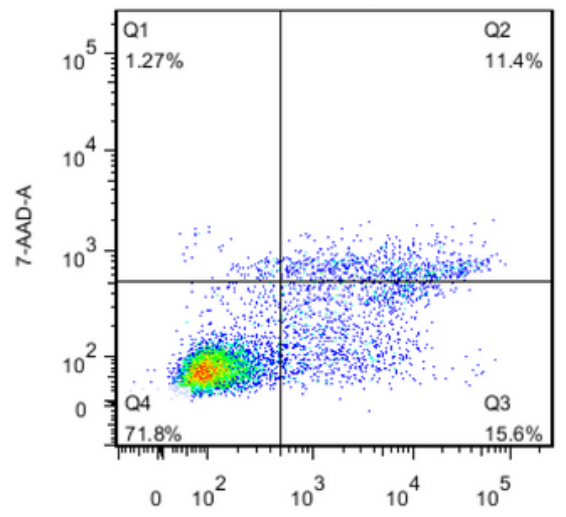

IL-1 $\beta$
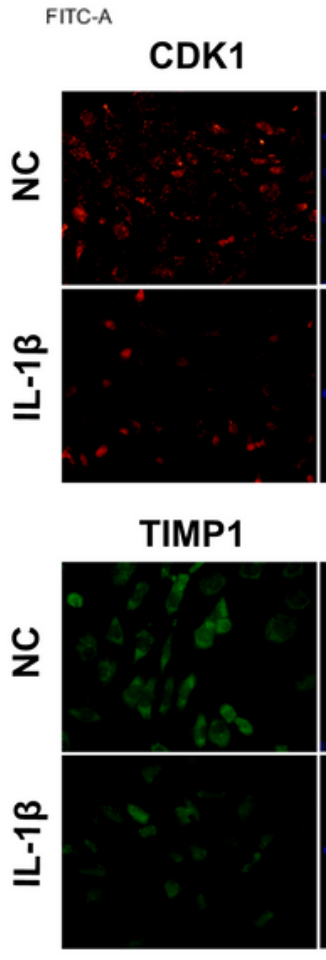

C
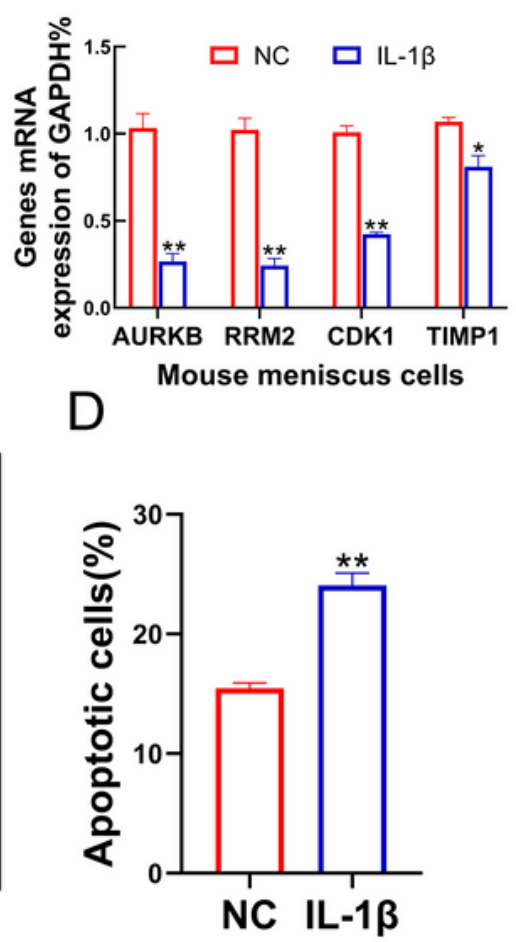

Figure 6 
The expressions of Hub genes in the mouse meniscus cells. (A) SA- $\beta$-gal staining of mouse senescent meniscus cells induced by IL-1 $13(10 \mathrm{ng} / \mathrm{ml})$ for $48 \mathrm{~h}$. Scale bar: $500 \mu \mathrm{m}$. (B and D) Apoptotic analysis detected by Annexin V/7AAD after IL-1 $\beta$ disposition. (C) Detection of the expressions of hub genes in senescent meniscus cells via qRT-PCR. We normalized the gene expression levels to GAPDH. (E) Immunofluorescence analysis of hub genes after treating meniscus cells with $10 \mathrm{ng} / \mathrm{ml} \mathrm{IL}-1 \beta$ for $48 \mathrm{~h}$. Scale bars, $50 \mu \mathrm{m}$. RRM2, ribonucleotide reductase regulatory subunit M2; AURKB, aurora kinase B; CDK1, cyclin dependent kinase 1; TIMP1, tissue inhibitor of metalloproteinases 1; GAPDH, Glyceraldehyde 3phosphate dehydrogenase. $n=6$ per group, mean \pm S.E.M., ${ }^{\star} P<0.05$, ${ }^{\star} \mathrm{P}<0.01$ in comparison with the control group.

\section{Supplementary Files}

This is a list of supplementary files associated with this preprint. Click to download.

- ARRIVEguidelines.pdf 